\title{
Du simple stand d'information au point de rencontre interactif
}

Cette année, la FMH a offert un nouveau concept de stand novateur. L'accent a été mis sur l'interaction et la rencontre avec les visiteurs. Par ailleurs, pour la première fois de leur histoire, la FMH, FMH Services, les éditions EMH et HIN ont tenu cette année un stand commun. Les responsables du stand tirent un bilan positif de cette expérience et relèvent l'effet de synergie qui a résulté de la collaboration entre ces quatre organisations.

\section{Maximilano Wepfer}

Chef suppléant de la division Communication de la FMH
Correspondance:

FMH / Division Communication Elfenstrasse 18

CH-3000 Berne 15

Tél. 0313591111

Fax 0313591112
Lors du salon spécialisé du marché de la santé (IFAS) 2014, le stand commun de la FMH, de FMH Services et de HIN n'est pas passé inaperçu. Les trois organisations ont en effet participé ensemble à l'IFAS qui s'est tenu du 21 au 24 octobre à Zurich. "Grâce à notre emplacement situé à côté de l'entrée, notre stand était incontournable», se réjouit Christoph Kreyden, responsable du stand et chef du Service d'administration des membres.

Christoph Kreyden, qui représente régulièrement la FMH lors de congrès et de foires depuis 2002, se remémore comment tout a commencé: «Au début, nous n'avions qu'un stand portable qu'il fallait monter nous-mêmes, mais au bout de quatre ans, nous nous étions tellement développés qu'il a fallu engager un monteur. Bien sûr, tout n'a pas été idéal tout
Présence confirmée des quatre prestataires dans les congrès et foires suivants en 2015

\begin{tabular}{lll} 
Manifestation & Date & Lieu \\
\hline Congrès JHaS & 18.4.2015 & Thoune \\
\hline $\begin{array}{l}\text { Assemblée annuelle } \\
\text { SSMI }\end{array}$ & $20.5 .2015-22.5 .2015$ & Bâle \\
\hline Congrès CMPR & $25.6 .2015-26.6 .2015$ & Lucerne \\
\hline Swiss Family Docs & $27.8 .2015-28.8 .2015$ & Berne \\
& &
\end{tabular}

de suite; en 2008 par exemple, les présentations sur écran plat n'ont pas eu l'effet spectaculaire escompté auprès des visiteurs. Au final, ce qui prime, c'est le contact direct avec le public», souligne Ch. Kreyden. «Lors des foires et des congrès, les membres de la

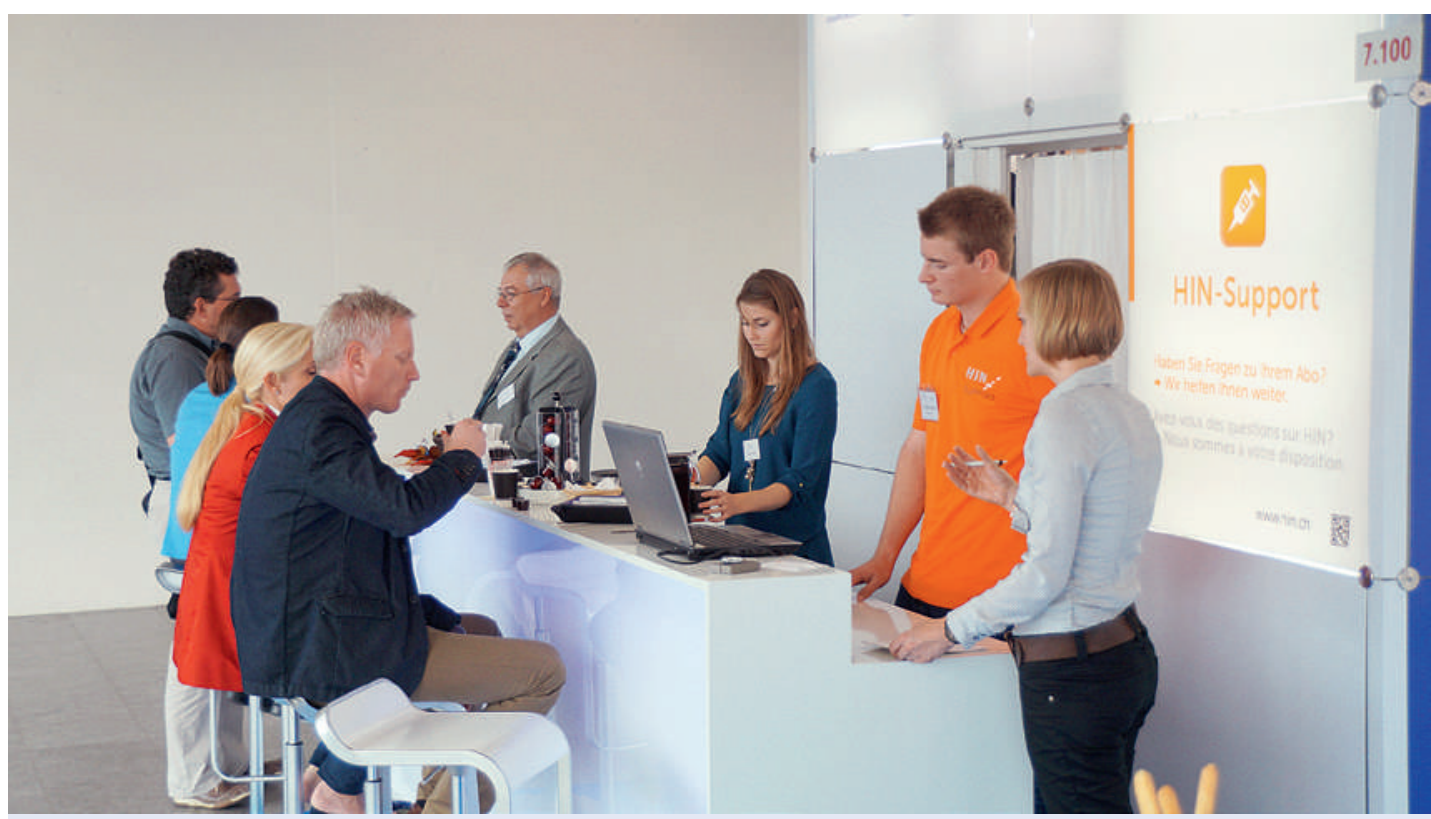

Les membres de la FMH bénéficient de conseils directs et compétents. 


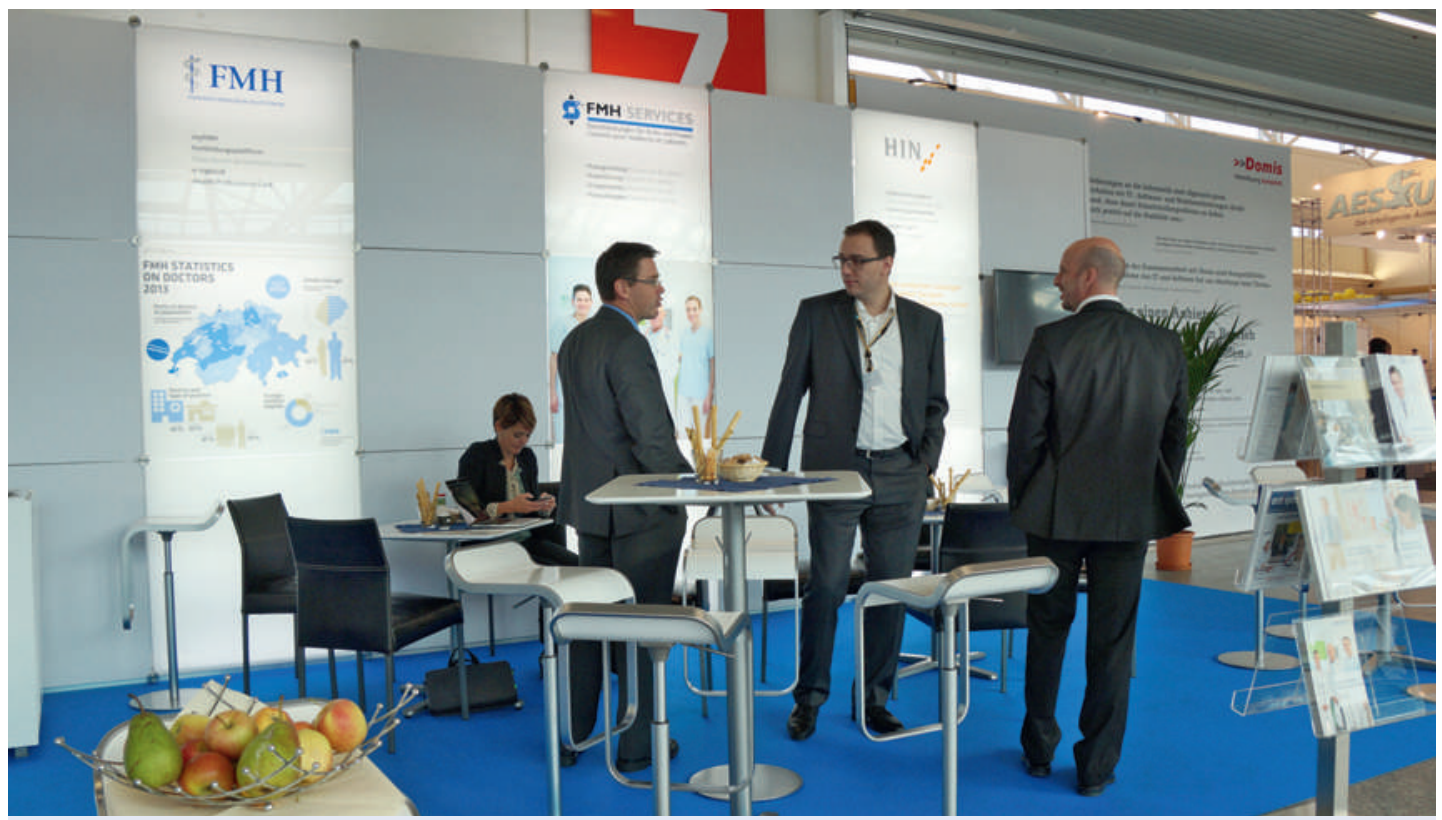

Le nouveau stand de la FMH, de FMH Services et de HIN offre de nombreuses possibilités de dialogue et d'interaction.

FMH peuvent découvrir les nombreuses prestations proposées dans différents domaines, notamment la gestion d'un cabinet médical, l'échange de données ou les publications médicales. Les visiteurs peuvent aussi directement évoquer leurs difficultés ou faire des suggestions, et nous pouvons leur venir en aide directement ou prendre acte de leur requête.»

En raison de l'importance de ce contact direct, il a été décidé l'année dernière de remanier complètement le concept du stand, car l'expérience a montré que l'ancienne configuration dressait une barrière inconsciente entre les visiteurs et les interlocuteurs de la FMH, laissant peu d'espace à la parole. «Nous avons donc voulu créer un stand qui ne soit plus purement informatif, mais qui devienne un point de rencontre», explique Ch. Kreyden. Ce nouveau stand a été inauguré cette année: sa structure est légère, il est plus aéré et lumineux et les nombreuses tables invitent le public à venir discuter. «Ainsi, nous pouvons mieux rencontrer le public, créer un contact plus personnel et offrir des conseils plus adaptés», se réjouit Ch. Kreyden. Grâce à sa structure modulaire, le stand s'adapte également mieux à la surface disponible pour chaque foire.
L'autre réflexion à la base de la création de ce nouveau stand a été, d'une part, le choix de FMH et FMH Services d'étendre leur collaboration aux EMH et à HIN et, de l'autre, le souci de mieux promouvoir

\section{Il est d'ailleurs prévu d'élargir encore l'offre et d'y inclure I'ISFM.}

leurs prestations. Ch. Kreyden apprécie les synergies qui résultent de cette collaboration. "Les médecins ont recours à ces quatre prestataires, s'ils peuvent profiter d'une offre groupée dans un même espace, c'est pertinent pour eux.» Il est d'ailleurs prévu d'élargir encore l'offre et d'y inclure l'ISFM, de sorte que les visiteurs puissent obtenir toutes les informations utiles sur la formation médicale postgraduée et continue. Ch. Kreyden espère que ce nouveau concept continuera à faire ses preuves à l'instar de cette première année d'essai et que son développement sera poursuivi au cours des années à venir. 